\title{
TRAUMATISMOS DE VEIA CAVA INFERIOR
}

\section{INFERIOR VENA CAVA INJURIES}

\author{
Cleinaldo de Almeida Costa, TCBC-AM¹; José Carlos Costa Baptista-Silva, TCBC-SP²; \\ Lana Márcia Esteves Rodrigues ${ }^{3}$; Fabiana Lo Presti Mendonça ${ }^{4}$; Thiago Silveira Paiva ${ }^{5}$; Emil Burihan
}

\begin{abstract}
RESUMO: Objetivo: Avaliar a incidência, o perfil clínico e as estratégias operatórias dos ferimentos de Veia Cava Inferior (VCI). Métodos: Foram analisados retrospectivamente os prontuários de 76 doentes com ferimento de VCI atendidos nos dois prontossocorros de Manaus, no período de janeiro de 1997 a julho de 2002. Mecanismo de lesão, mortalidade, estado hemodinâmico, índice de trauma abdominal penetrante (PATI), achados intra-operatórios e conduta cirúrgica foram estudados. Resultados: Quarenta e nove $(65 \%)$ doentes sofreram lesão por arma branca, 26 (34\%) por arma de fogo e um por traumatismo abdominal fechado. Quarenta e um (54\%) doentes sobreviveram. Quase todos chegaram acordados, entretanto $40 \%$ estavam hipotensos (pressão arterial sistólica $<70 \mathrm{mmHg}$ ). O índice de trauma abdominal penetrante (PATI) médio foi maior que 40. À laparotomia, todos demonstraram sangramento retroperitoneal ativo ou hematoma retroperitoneal em expansão. Vinte e um pacientes possuíam lesão de VCI retro-hepática, enquanto nos outros 55 a lesão era infra-hepática. O reparo operatório predominante foi a venorrafia lateral em 65 doentes. Houve necessidade de tóraco-freno-laparotomia em quatro doentes do total de oito doentes que foram à toracotomia direita por lesão retro-hepática. Foram realizados shunts átrio-cavais em seis doentes, dos quais três sobreviveram. Conclusões: O ferimento de VCI é uma lesão de alta letalidade e possui uma relação intrínseca com a violência urbana. A sobrevivência depende de uma imediata e vigorosa reposição de volume, um manejo operatório adequado e todo esforço em evitar-se hipotermia (Rev. Col. Bras. Cir. 2005; 32(5): 244-250).
\end{abstract}

Descritores: Veia Cava Inferior; Veia cava inferior /lesões; Ferimentos penetrantes.

\section{INTRODUÇÃO}

A lesão vascular é causa primária de morte no traumatismo abdominal. Qualquer doente com ferimento abdominal chegando in extremis deve ser considerado como portador de uma lesão vascular abdominal ${ }^{1}$. Particularmente graves, as lesões venosas abdominais maiores têm uma mortalidade entre 50 e $90 \%{ }^{2,3}$, e tornam necessário um conhecimento abrangente dos diversos padrões de lesões vasculares abdominais e das múltiplas estratégias operatórias disponíveis ${ }^{4}$.

Hoje, as lesões vasculares são um problema demasiadamente comum nos centros de trauma urbanos, e ocorrem em 10 a 20\% de todos os doentes submetidos à laparotomia por traumatismos penetrantes na população civil urbana ${ }^{5-13}$.

Analisando 104403 necrópsias no Instituto Médico Legal de São Paulo de 1984 a 1993, Delmonte Fernandes ${ }^{14}$ encontrou 153 casos de lesão vascular, observando que deste total 45 óbitos ocorreram por ferimentos de Veia Cava Inferior (VCI) $(29,4 \%)$.

No Amazonas, de acordo com dados da Secretaria Estadual de Saúde, foram atendidos cerca de oito mil doentes com traumatismos graves no período de 1997 a 1999, cujo repasse do Sistema Único de Saúde (SUS), incluindo tratamento cirúrgico e unidade de terapia intensiva, pode chegar a $\mathrm{R} \$ 2533,57$ por cada doente individualmente ${ }^{15}$.

O presente estudo tem o objetivo de avaliar a incidência de ferimentos de VCI atendidos na cidade de Manaus, no período de janeiro de 1997 a julho de 2002, além de observar o tratamento empregado e os principais aspectos relacionados à morbidade $\mathrm{e}$ mortalidade destes traumatismos.O Protocolo deste estudo retrospectivo foi aprovado pela Comissão de Ética Médica do Hospital Universitário Getúlio Vargas e pelo Comitê de Ética Universidade Federal de São Paulo UNIFESP-EPM.

\section{MÉTODO}

O Pronto Socorro Municipal 28 de Agosto e Hospital Pronto Socorro João Lúcio Pereira Machado são as duas unidades do Sistema Único de Saúde que atendem a totalidade dos casos de traumatismos graves em adultos na cidade de Manaus.

Um estudo retrospectivo abrangendo 67 meses, de janeiro de 1997 a julho de 2002, analisou 76 casos de

1. Professor da disciplina de Cirurgia Vascular da Universidade do Estado do Amazonas; Mestre em Cirurgia Vascular pela Universidade Federal de São Paulo; Especialista em Cirurgia Vascular pela SBACV.

2. Professor Associado (Livre Docente) da disciplina de Cirurgia Vascular do Departamento de Cirurgia da Universidade Federal de São Paulo.

3. Chefe de Cirurgia Gastroenterológica do Hospital Universitário Dona Francisca Mendes - Universidade Federal do Amazonas.

4. Residência em Cirurgia Vascular realizada no Hospital da Força Aérea do Galeão - Rio de Janeiro.

5. Residência em Cirurgia Geral realizada no Hospital Universitário Getúlio Vargas - Universidade Federal do Amazonas.

6. Professor Titular, Coordenador do Convênio Interinstitucional entre a Universidade Federal de São Paulo e a Universidade Federal do Amazonas.

Recebido em: 03/03/2005

Aceito para publicação em: 26/09/2005

Conflito de Interesse: nenhum

Fonte de financiamento: nenhuma

Trabalho realizado no Hospital Pronto-Socorro Dr. João Lúcio Pereira Machado e no Pronto-Socorro Municipal 28 de Agosto, Manaus - Amazonas. 
traumatismos de VCI atendidos nos dois prontos-socorros de Manaus neste período. Foram incluídos no estudo todos os doentes admitidos vivos e nos quais, durante a laparotomia exploradora, ficou evidenciado o ferimento de VCI.

Os doentes foram atendidos pelas múltiplas equipes que compõem as escalas de plantões dos dois prontos-socorros. Além de dados como idade, sexo, mecanismo de trauma e local da lesão, foram relacionadas as lesões abdominais associadas, agrupadas de acordo com o Penetrating Abdominal Trauma Index (PATI) ${ }^{16}$. Este índice prevê o registro de lesões existentes no duodeno, pâncreas, fígado, intestino delgado e grosso, vias biliares extra-hepáticas, estômago, baço, rins, ureteres, bexiga, grandes vasos, pequenos vasos e ossos. $\mathrm{O}$ cálculo do resultado final e sua correlação com o valor prognóstico estima que a maior gravidade corresponde a índices superiores a $25^{16,17}$.

Foi avaliada a presença de choque hipovolêmico à admissão, conforme definido pelo programa ATLS, que classifica a perda estimada de líquidos e sangue em classes, relacionadas a dados de frequiência de pulso, pressão arterial e diurese, entre outros ${ }^{18}$.

Outras variáveis incluíram o tempo decorrido entre a admissão no pronto-socorro e o início da cirurgia, a condição de integridade do retroperitônio, a conduta cirúrgica adotada para cada doente, o tempo de internação, as complicações e a mortalidade.

As soluções utilizadas para reanimação e durante a cirurgia foram os cristalóides (soro fisiológico 0,9\%, ringer

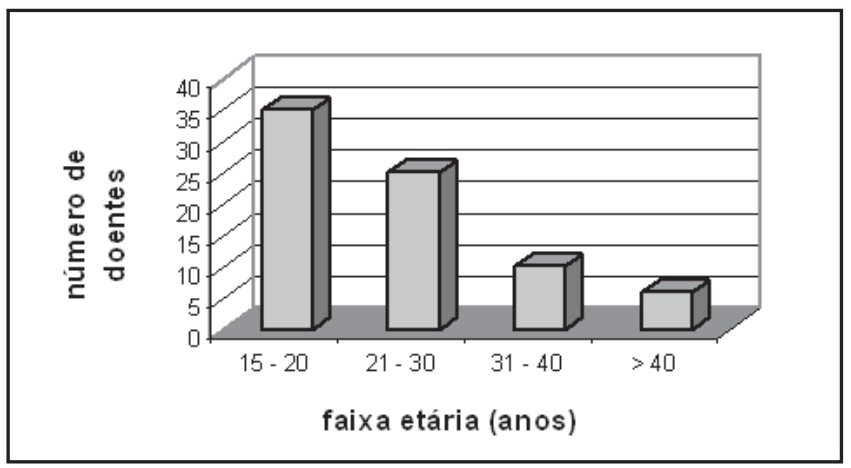

Figura 1-Representação gráfica dos doentes, segundo a faixa etária.

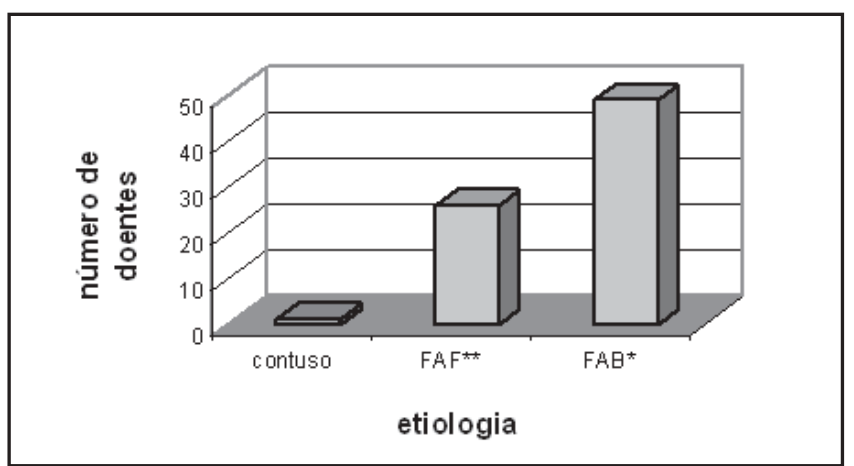

Figura 2 - Representação gráfica da etiologia.

\footnotetext{
* Ferimento por arma branca

** Ferimento por arma de fogo
}

com lactato) e hemoderivados, previamente aquecidos a $37^{\circ} \mathrm{C}$.

Outros exames utilizados durante o atendimento inicial constaram de medida do pulso e pressão arterial, hematócrito, hemoglobina, tipagem sangüínea e prova cruzada para todos os doentes, teste de gravidez nas doentes em idade fértil, radiografias de tórax e bacia em posição ânteroposterior. Todos os doentes foram submetidos ao cateterismo vesical e quantificação do débito urinário.

Inicialmente foi realizada uma análise descritiva dos dados epidemiológicos, e para estudar a relação entre as variáveis óbito, posição da lesão na VCI, pressão arterial sistólica e hematócrito, foi utilizado o teste do Qui-quadrado, com nível de significância de 5\%.

Para verificar se houve relação entre a variável óbito e o Penetrating Abdominal Trauma Index (PATI) foi utilizado o teste de aderência Kolmogorov-Smirnov com nível de 5\% de significância, que trata dos dados individualmente.

A seguir, fez-se uma comparação de médias entre a variável óbito, utilizando-se para tanto o teste não paramétrico de Wilcoxon, com nível de 5\% de significância, com as variáveis: 1-cristalóides, 2-hemoderivados, 3-hematócrito e 4-PA sistólica.

\section{RESULTADOS}

Dos 76 doentes, 41 (54\%) sobreviveram. Os traumatismos de VCI ocorreram em 35 doentes entre 15 e 20 anos (46\%), 25 entre 21 e 30 anos (33\%), dez doentes entre 31 e 40 anos (13\%) e seis com mais de 40 anos (8\%) (Figura 1).

Houve predomínio de doentes do sexo masculino, com 69 doentes em 76 (91\%). Verificou-se entre os doentes estudados que a maior incidência de traumatismo de VCI foi ocasionada por arma branca (FAB), com 49 doentes (65\%), seguida por arma de fogo (FAF) com 26 doentes (34\%) e um caso de traumatismo abdominal contuso (1\%) (Figura 2).

Ao analisar a incidência de ferimentos de VCI a cada ano, observou-se um aumento de $160 \%$ nos ferimentos por FAF em 1999 (passando de cinco para 13), quando o número de ferimentos de VCI por arma de fogo ultrapassou o número de ferimentos por arma branca (Tabela 1).

A análise da relação entre a mortalidade e o agente vulnerante mostrou não haver diferença estatisticamente significativa $(\mathrm{p}=0,236)$ entre os ferimentos produzidos por arma branca e os produzidos por arma de fogo (Tabela 2).

Vinte e três dos 35 doentes que morreram $(65,7 \%)$ foram operados em um tempo inferior a 60 minutos. Nove doentes dos 41 sobreviventes foram levados à cirurgia em igual período de tempo.

Houve oito lesões associadas de VCI e aorta, com apenas um sobrevivente.

Dos doentes que morreram, $27(77,1 \%)$ apresentaram pressão arterial sistólica inferior a $70 \mathrm{mmHg}$ à admissão e 19 (70,3\%) tinham hematócrito inicial menor que $28 \%$.

Observou-se um consumo médio de 3,9 litros de soluções cristalóides pelos sobreviventes, contra 5,1 litros pelos doentes que foram a óbito e 1,0 litro de hemoderivados contra 4,5 litros respectivamente. 
Tabela 1 - Distribuição segundo o número de doentes por ano, com relação à etiologia.

\begin{tabular}{ccccc}
\hline Ano & Etiologia & & Total \\
& FAB $^{*}$ & FAF** & Contuso & \\
\hline 1997 & $9(60,0 \%)$ & $5(33,33 \%)$ & $1(6,67 \%)$ & $15(100,0 \%)$ \\
1998 & $13(72,22 \%)$ & $5(27,78 \%)$ & $0(0 \%)$ & $18(100,0 \%)$ \\
1999 & $11(46 \%)$ & $13(54 \%)$ & $0(0 \%)$ & $24(100,0 \%)$ \\
2000 & $8(89 \%)$ & $1(11 \%)$ & $0(0 \%)$ & $9(100,0 \%)$ \\
2001 & $4(80 \%)$ & $1(20 \%)$ & $0(0 \%)$ & $5(100,0 \%)$ \\
2002 & $4(80 \%)$ & $1(20 \%)$ & $0(0 \%)$ & $5(100,0 \%)$ \\
Total & $49(64 \%)$ & $26(34 \%)$ & $1(2,0 \%)$ & $76(100,0 \%)$ \\
\hline
\end{tabular}

* Ferimento por arma branca

** Ferimento por arma de fogo

Tabela 2 - Relação entre a mortalidade e o agente vulnerante.

\begin{tabular}{lccc}
\hline Agente & \multicolumn{2}{c}{ Óbito } & Total \\
& Não & Sim & \\
\hline FAB & $28(57,14 \%)$ & $21(42,86 \%)$ & $49(100,0 \%)$ \\
FAF & $12(46,15 \%)$ & $14(53,84 \%)$ & $26(100,0 \%)$ \\
Total & $40(53,33 \%)$ & $35(46,67 \%)$ & $75(100,0 \%)$ \\
\hline
\end{tabular}

*O teste utilizado foi o exato de Fisher. $p=0,236$.

Não houve avaliação pré-operatória por tomografia computadorizada de abdome e não foi realizado tratamento não-operatório de VCI nos doentes deste estudo.

Houve necessidade de toracotomia à direita em cinco sobreviventes e em três doentes que morreram, incluídas nestes números quatro toracofrenolaparotomias.

Foi realizada ligadura de VCI infra-renal em três doentes, com apenas um sobrevivente.

Utilizamos shunt átrio-caval somente em seis doentes, com três óbitos (Figura3).

Quatorze doentes morreram durante a cirurgia (18\%), 18 pacientes em menos de 24 horas após o atendimento (24\%) e três pacientes (3\%) não sobreviveram a complicações durante a primeira semana após o trauma. Portanto todos os óbitos ocorreram no primeiro mês de pós-operatório (Figura 4).

O mesogástrio foi o local de lesão atingido em 16 doentes (21\%), seguido de epigástrio e hipocôndrio direito, com 14 e 12 (19\% e 16\%) doentes respectivamente.

Do total de 76 doentes, 55 (72\%) apresentaram lesão de VCI infra-hepática e 21 (28\%) apresentaram lesão de VCI retro-hepática.

Evidenciou-se hematoma retroperitoneal em 41 doentes (54\%). Destes 30 sobreviveram (Tabela 3).

Do total de 41 sobreviventes, $31(75,6 \%)$ tinham na admissão, hematócrito superior a 28\%, e 32 (78\%) apresentavam pressão arterial sistólica inicial superior a $70 \mathrm{mmHg}$.

O Penetrating Abdominal Trauma Index (PATI) apresentou a média de 38,55 nos sobreviventes, enquanto nos que morreram a média foi de 48 (Tabela 4). A Tabela 4 também sintetiza os principais parâmetros clínicos e reposição de volume, com relação à mortalidade.

\section{DISCUSSÃO}

Os ferimentos penetrantes de VCI continuam representando um problema grave e um desafio para os cirurgiões em atividade nos prontos-socorros ${ }^{18}$. Nas últimas três décadas foram desenvolvidos centros de trauma e sistemas integrados de atendimento ao doente vítima de traumatismos, culminando na remoção mais segura e transporte rápido, além da significativa mudança de mentalidade e sistematização no atendimento inicial do politraumatizado durante a primeira hora. Todavia, a mortalidade por ferimentos de VCI pouco se modificou ${ }^{19,20}$. A taxa de mortalidade após ferimentos de VCI em importantes séries manteve-se entre $33 \%$ e $66 \%$, atestando a gravidade deste tipo de lesão ${ }^{6,21}$. A mortalidade encontrada neste estudo foi de $46 \%$.

Vários autores acreditam que o desenvolvimento dos sistemas integrados de trauma possibilitou a chegada ao pronto-socorro de muitos doentes gravemente feridos e agonizantes, causando com isto a diminuição da mortalidade no local da ocorrência, mas, em contrapartida, aumentando a taxa de mortalidade intra-hospitalar ${ }^{19,20}$.

Tem sido consenso na literatura que a diminuição do tempo entre a admissão e o início da cirurgia, o emprego de protocolos agressivos de reanimação no doente gravemente ferido e recursos materiais e humanos adequados devem contribuir favoravelmente com a diminuição da mortalidade ${ }^{6,14}$.

Em Manaus, capital do estado do Amazonas, com população urbana estimada em um milhão e meio de habitantes, contamos apenas com dois prontos-socorros, mantidos pelo Sistema Único de Saúde (SUS), onde é atendida a totalidade dos casos de traumatismos graves ocorridos na cidade. O resgate é feito por pessoal paramédico do sistema municipal S.O.S. 192 e o atendimento cirúrgico hospitalar têm sido feito conforme o programa suporte avançado de vida no trauma ATLS, desde 1996.

Neste estudo os ferimentos de VCI produzidos por arma branca (FAB) foram claramente predominantes em $65 \%$ dos doentes, seguidos de ferimentos por projetis de arma de fogo (FAF) com $34 \%$ e traumatismo abdominal contuso em $1 \%$. Estes dados diferem bastante da literatura nacional e internacional ${ }^{5-7,14}$, na qual os FAF são bem mais freqüentes, mostrando uma peculiaridade regional, que se confirma com 


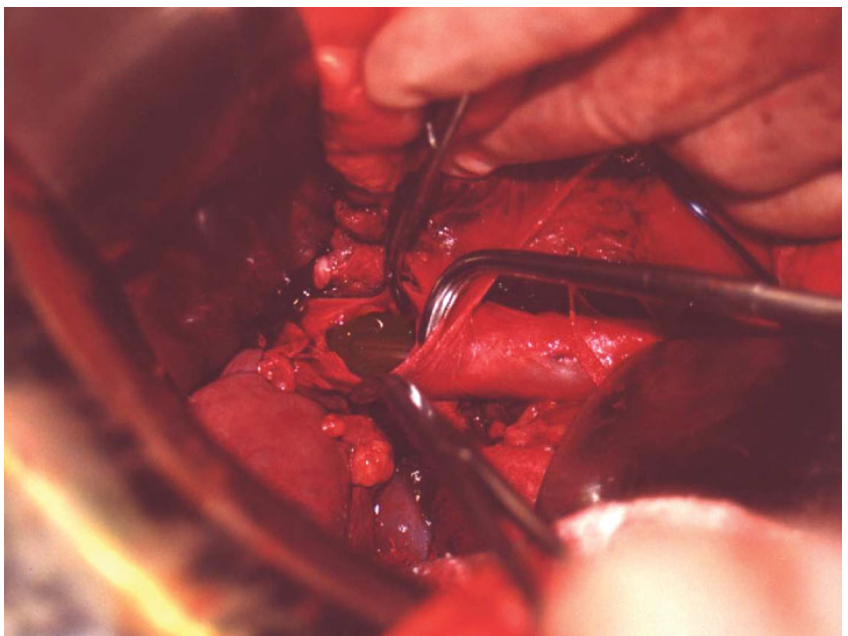

Figura 3 - Extenso ferimento de veia cava inferior retro-hepática, com shunt átrio-caval em seu interior (arquivo pessoal).

os dados da Secretaria Estadual de Segurança Pública, que relaciona $5840 \mathrm{FAB}$ seguidos de $2267 \mathrm{FAF}$, no período de 1997 à $1999^{15}$.

As lesões vulnerantes produzidas por instrumentos cortantes (facas, estiletes, vidro etc), apesar de poderem causar lesões extensas, geralmente não determinam perda de substância da parede venosa, sendo o reparo mais simples de ser realizado e a mortalidade menor ${ }^{20,21}$. Os FAF podem resultar em lacerações extensas, muitas vezes associadas a perdas significativas da parede venosa e comportam mortalidade significativa. No entanto, em nossa pesquisa não foi possível confirmar as afirmações concernentes à maior gravidade dos traumatismos de VCI produzidos por armas de fogo.

Vários autores situaram os traumatismos fechados ou não-penetrantes resultando em cerca de um a $10 \%$ de todos os traumatismos abdominais. Neste estudo encontramos apenas um caso de lesão contusa de VCI, produzida por acidente automobilístico ${ }^{6,9,10}$

Burihan $^{22}$ referiu-se à lesão iatrogênica dos grandes vasos abdominais como um problema incomum mas persistente. O fato de não termos encontrado nenhuma lesão iatrogênica não diminui sua possibilidade.

Leppaniemi, et al. ${ }^{23}$ chamaram atenção para o tempo decorrido entre a chegada ao hospital e o início da cirurgia, ou seja, o tempo consumido no diagnóstico e nas manobras de reanimação, e demonstraram que houve uma diferença nítida

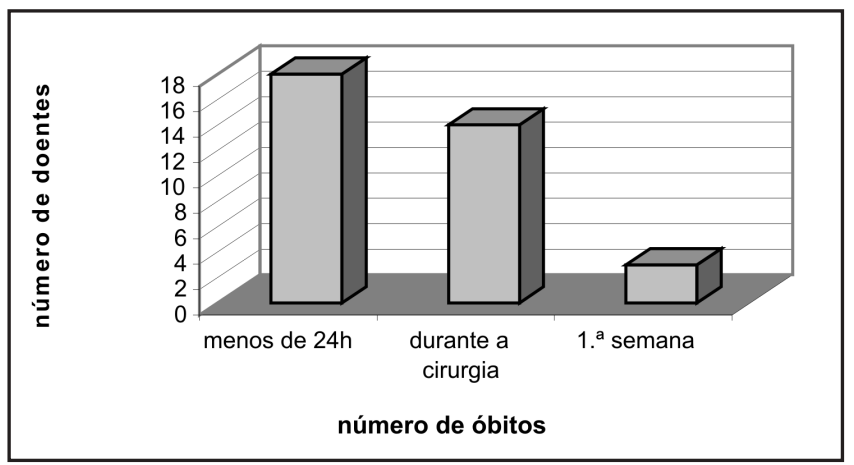

Figura 4 - Representação gráfica do número de óbitos. em relação ao tempo entre os doentes que morreram e os que sobreviveram.

Foi possível correlacionar o estado crítico do doente à maior rapidez na intervenção, ao constatarmos que 23 dos 35 doentes que morreram $(65,7 \%)$ foram operados em um tempo inferior a 60 minutos, a contar do momento de sua admissão. Em contrapartida, apenas nove doentes dos 41 que sobreviveram (21\%) foram levados à cirurgia em igual período de tempo.

As lesões arteriais estão associadas aos ferimentos de VCI como um fator agravante de letalidade. Coimbra et al. ${ }^{21}$ citaram que dentre nove doentes nesta condição apenas um sobreviveu. Encontramos oito lesões associadas de VCI e aorta, com apenas um sobrevivente.

Diversos trabalhos destacaram variáveis que tiveram impacto negativo na evolução dos doentes com traumatismos de VCI, entre estas a hipotensão arterial ao início da reanimação. Klein et al. ${ }^{20}$ referiram-se a oito doentes que morreram, todos com pressão arterial sistólica inferior a 80 $\mathrm{mmHg}$, entretanto não observaram diferença significativa no hematócrito em relação aos doentes que sobreviveram.

Dos doentes que morreram, $27(77,1 \%)$ apresentavam pressão arterial sistólica inferior a $70 \mathrm{mmHg}$ à admissão, sendo que 19 destes $(70,3 \%)$ tinham o hematócrito inferior a $28 \%$.

Estes dados clínicos observados à admissão nos remetem a outro importante fator prognóstico: a integridade do retroperitônio servindo de tamponamento para a lesão de VCI e garantindo maior estabilidade hemodinâmica ao doente ${ }^{14,20,21}$. Em nosso estudo verificamos que 24 doentes (69\%) dos 35 que foram a óbito não possuíam integridade retroperitoneal.

$\mathrm{O}$ choque hipovolêmico à admissão, secundário à ausência de tamponamento retroperitoneal e hemorragia abundante, além da incapacidade de recuperação dos parâmetros hemodinâmicos após a reposição volêmica são elementos de grande valor preditivo em relação ao prognóstico. Elementos estes que se confirmaram em nosso estudo.

Alguns trabalhos compararam o consumo de hemoderivados e soluções cristalóides, e puderam mostrar uma diferença significativa entre os grupos de sobreviventes e óbitos, com nítido predomínio destes ${ }^{14,20}$.

Neste trabalho comparando os grupos sobreviventes e óbitos, observamos um consumo médio de soluções cristalóides de 3,9 litros (L) contra 5,1 1 e de hemoderivados de 1,0L contra 4,5L. Nestas duas variáveis estudadas houve diferença estatisticamente significativa.

O PATI é o índice de trauma abdominal em uso atualmente nos dois prontos-socorros de Manaus. Muitos autores o consideraram superior ao Injury Severity Score (ISS) para previsão de mortalidade ${ }^{6,16,17,23}$. Sua pontuação acima de 25 prediz uma mortalidade superior a $44 \%$.

Avaliando os grupos de sobreviventes e óbitos obtivemos uma média de PATI de 38,5 e 48 respectivamente, o que indicaria mortalidade entre 47 e $50 \%$ para toda a amostra. Como a mortalidade encontrada foi de $46 \%$, situamo-nos próximos da média relatada na literatura.

Leppaniemi et al. ${ }^{23}$ reportaram o uso restrito de métodos sofisticados de imagem, para casos cuidadosamente 
Tabela 3 - Correlação entre a presença de hematoma retroperitoneal e mortalidade.

\begin{tabular}{|c|c|c|c|}
\hline \multirow[t]{2}{*}{ Mortalidade } & \multicolumn{2}{|c|}{ Hematoma retroperitoneal } & \multirow[t]{2}{*}{ Total } \\
\hline & Ausência & Presença & \\
\hline Sobrevivente & $11(27 \%)$ & $30(73 \%)$ & $41(100,0 \%)$ \\
\hline Óbito & $24(69 \%)$ & $11(31 \%)$ & $35(100,0 \%)$ \\
\hline Total & $35(46 \%)$ & $41(54 \%)$ & $76(100,0 \%)$ \\
\hline
\end{tabular}

* teste do Qui-quadrado significativo - $p=0,001$.

selecionados de doentes hemodinamicamente estáveis, que uma vez conduzidos à tomografia computadorizada de abdome, tiveram confirmado o diagnóstico de lesão de VCI. Rozycki et al. ${ }^{24}$ referiram-se ao uso da ultra-sonografia na sala de emergência, como um recurso rápido e não-invasivo para a avaliação de hemoperitônio ou hematoma retroperitoneal.

Poucos recursos de imagem foram documentados em nosso trabalho. Nenhum de nossos doentes foi avaliado por meio de tomografia computadorizada e não houve nenhum doente com ferimento de VCI conduzido sem tratamento operatório. $\mathrm{O}$ alto número de lesões abdominais penetrantes (75 doentes em 76) associado ao quadro hemodinâmico foram determinantes para orientar a decisão operatória.

Em todos os doentes que fizeram parte deste estudo utilizamos incisão mediana xifopubiana, universalmente aceita no manejo dos ferimentos vasculares abdominais.

Houve necessidade de toracotomia à direita em cinco sobreviventes e em três doentes que morreram, além de quatro toracofrenolaparotomias, o que confirmou a abertura torácica nos ferimentos de VCI em $10 \%$ de nossa casuística.

O tratamento dos ferimentos de VCI obedece aos princípios básicos de exposição controle e reparo da lesão ${ }^{25,26}$.

A sangria proveniente de uma laceração da VCI pode ser temporariamente controlada por compressão manual até uma completa exposição, seguida de pinçamento proximal e distal e correção do ferimento por meio de sutura vascular com fio de polipropileno 4-0, procedimento adotado em $85 \%$ dos pacientes. Em caso de lesão de parede posterior, a venorrafia é possível por meio de delicada rotação, tomandose todo o cuidado com as veias lombares, ou ampliando-se a lesão anterior ${ }^{14,26}$.

Coimbra et al. ${ }^{21}$ desencorajaram o uso de enxertos sintéticos devido a altas taxas de infecção e trombose. No entanto, alguns autores descreveram o uso do politetrafluoretileno (PTFE) com boas taxas de permeabilidade na reconstrução da $\mathrm{VCI}^{5,6}$.

A ligadura da VCI infra-renal pode ser realizada, particularmente em doentes com choque hipovolêmico, politransfundidos e com coagulopatia ${ }^{14,26}$. O ideal é o reparo de todas as lesões venosas, entretanto isto pode não ser absolutamente necessário. Um tratamento seletivo, refletindo o mecanismo de trauma, a magnitude da perda sangüínea, o tempo de anestesia, lesões concomitantes de outros órgãos, extensão da lesão venosa e duração da isquemia podem ser amenizados em contraposição a um tempo maior consumido no reparo venoso ${ }^{27}$. O procedimento de ligadura da VCI infrarenal pode levar a edema bilateral de membros inferiores, varizes secundárias e úlceras de estase venosa em até um terço dos doentes ${ }^{26}$.

Em nosso estudo três doentes foram submetidos à ligadura da VCI infra-renal, com um sobrevivente. Apesar de estar hoje estabelecida como uma manobra prudente para o doente crítico, a ligadura também foi pouco utilizada em várias séries ${ }^{19}$.

Foi relatado o uso da ligadura da veia renal esquerda para possibilitar a mobilização da VCI na confluência das veias renais. Entretanto, Baptista-Silva et al ${ }^{27}$ demonstraram experimentalmente acentuadas alterações patológicas e complicações renais, testiculares e das glândulas supra-renais após a ligadura da veia renal esquerda e enfatizaram que este é um procedimento de alto risco, devendo ser evitado.

Muitos autores citaram que a lesão de VCI em sua porção retro-hepática comporta taxas mais altas de mortalidade comparada com as lesões das outras porções desse vaso $^{4,5,14}$. Estas lesões, independentemente do agente vulnerante, são situações complexas e desafiadoras devido à hemorragia abundante, associação de lesões em outros órgãos e pela complexidade do acesso operatório ${ }^{28}$. Várias proposições surgiram para a dissecção das lesões de VCI retrohepática, desde 1- dissecção direta da lesão, 2- a utilização de shunts intra-cava, até 3- dissecção trans-hepática. A respeito do shunt átrio-caval, Nance ${ }^{28}$ comentou que "semelhante a galinha que teve o seu pescoço torcido e ainda sobrevive, o shunt átrio-caval ainda persiste". E Degiannis et al. ${ }^{18}$ afirmou que "muito tem sido escrito sobre lesões venosas hepáticas e é possível que haja mais autores sobre esse assunto que sobreviventes aos procedimentos descritos".

Tabela 4 - Comparação de médias entre os dados clínicos com relação à mortalidade.

\begin{tabular}{lccccc}
\hline Parâmetros Laboratoriais & \multicolumn{2}{c}{ Sobreviventes } & \multicolumn{2}{c}{ Óbitos } & $*$ P-valor \\
& Médias & Desvio-padrão & Médias & Desvio-padrão & \\
\hline PATI** & 38,545 & 9,936 & 48,000 & 11,944 & 0,0117 \\
PAS*** (mmHg) & 98,966 & 26,260 & 53,000 & 28,991 & 0,0001 \\
Hematócrito (vol\%) & 32,193 & 6,988 & 25,965 & 9,650 & 0,0059 \\
Cristalóides (L) & 3,900 & 1,093 & 5,100 & 1,561 & 0,0019 \\
Hemoderivados (L) & 1,000 & 1,351 & 4,575 & 2,834 & 0,0001 \\
\hline
\end{tabular}

* se p-valor menor que 0,05 existe diferença estatisticamente significativa.

** Penetrating Abdominal Trauma Index.

*** Pressão arterial sistólica (em milímetros de mercúrio). 
De fato, Kudsk et al. ${ }^{29}$ revisaram os seis principais relatos de uso do shunt átrio-caval (onde a maior série tinha cinco doentes) e encontraram 13 óbitos em 18 doentes, uma mortalidade de (72\%). Encontramos 21 doentes com lesão de VCI retro-hepática, $76 \%$ dos quais foram a óbito. Utilizamos o shunt somente em seis oportunidades, com três óbitos. Tão pequena experiência não nos permite tecer considerações quanto à sua validade. Entretanto é justo lembrar que, ao deparar-se com lesão de VCI retro-hepática, o cirurgião encontra-se em uma situação limite, altamente desafiadora, onde o conhecimento de manobras críticas no trauma somado à experiência poderão ser de muita valia.

Ainda que existam diversos métodos operatórios descritos para os ferimentos de VCI em suas várias localiza- ções, pensamos que a melhor técnica é aquela com a qual o cirurgião está mais familiarizado.

O traumatismo de VCI continua sendo uma situação desafiadora. Trata-se de uma doença de população jovem, urbana, predominantemente masculina e fundamentalmente relacionada à violência interpessoal. Em Manaus são atendidos em média doze doentes por ano, vítimas deste tipo de lesão e apesar dos avanços obtidos na atenção pré-hospitalar e intra-hospitalar o traumatismo de VCI continua representando um problema cirúrgico de alta mortalidade (46\% destes doentes). Como problema de saúde pública é uma doença evitável (como todo trauma), onde a educação e leis que inibam a posse e o porte de armas podem fazer uma enorme diferença.

\begin{abstract}
Background: Injuries of inferior vena cava (IVC) require immediate and definitive action. Our objective is to evaluate the incidence, the clinical findings and the operative approach to IVC injuries. Methods: We retrospectively studied 76 patients with IVC injuries treated in two Emergency Hospitals of Manaus, AM, Brazil, from January 1997 to July 2002. Mechanisms of injuries, mortality, hemodynamic status, penetrating abdominal trauma index (PATI), intraoperative findings and surgical approach were among assessed data. Results: Fourty-nine patients (65\%) had stab wounds, 26(34\%) gunshot wounds, and one had blunt abdominal trauma. Fourty-one (54\%) patients survived. Almost all patients entered the emergency room awake, although $40 \%$ were hypotensive (systolic blood pressure $<70 \mathrm{mmHg}$ ), and the penetrating abdominal trauma index average was above 40. At laparotomy, active retroperitoneal bleeding or an expanding retroperitoneal hematoma were detected in all cases. Caval injury was retro-hepatic in 21 patients, and infrahepatic in the other 55. The prevailing surgical approach was lateral repair in 65 patients. Atrial-caval shunting was tried in six patients, with only three survivals. Conclusions: We concluded that IVC injury remains a highly lethal injury and there is a close relationship with urban violence. This study emphasizes that successful outcome depends on prompt volume restoration, avoidance of hypothermia and a stratified selective surgical approach.
\end{abstract}

Key Words: Vena cava, inferior; Vena cava, inferior/injuries; Wounds, penetrating.

\section{REFERÊNCIAS}

1. Rummel MC, Kerstein MD, Palacios, GM. Trauma vascular abdominal. In: Rodriguez A, Ferrada R, editores. Trauma. Bogotá: Impresora Feriva; 1997. p. 411-26.

2. Feliciano DV, Burch JM, Spjut-Patrinely V, et al. Abdominal gunshot wounds. An urban trauma center's experience with 300 consecutive patients. Ann Surg. 1988; 208(3):362-70.

3. Feliciano DV. Management of traumatic reproperitoneal hematoma. Ann Surg. 1990; 211(2):109-23.

4. Kashuk JL, Moore EE, Millikan JS, et al. Major abdominal vascular trauma - a unified approach. J Trauma. 1982;22(8): 672-9.

5. Feliciano DV, Burch JM, Graham JM. Abdominal vascular injury. In: Feliciano DV, Moore EE, Mattox KL, editors. Trauma. Stanford: Appleton \& Lange; 1999. p. 615-33.

6. Mattox KL, Feliciano DV, Burch J, et al. Five thousand seven hundred sixty cardiovascular injuries in 4459 patients. Epidemiologic evolution 1958 to 1987. Ann Surg. 1989;209(6):698-707.

7. Caps MP. The epidemiology of vascular trauma. In: Rutherford $R B$, editor. Vascular Surgery. $5^{\text {th }}$ ed. Philadelphia: WB Saunders Company; 2000. p. 857-62.

8. Melzer-Lange MD. Violência associada a comportamento de alto risco para a saúde em adolescentes. In: Hennes H, Calhoun AD.
Clínicas Pediátricas da América do Norte. Rio de Janeiro: Interlivros Edições; 1998. p. 43-55.

9. Cox EF. Blunt abdominal trauma. A 5-year analysis of 870 patients requiring celiotomy. Ann Surg. 1984;199(4):467-74.

10. Cohen D, Johansen K, Cottingham K, et al. Trauma to major visceral veins: an underemphasized cause of accident mortality. J Trauma. 1980;20(11):928-32.

11. Mattox KL, McCollum WB, Jordam GL, et al. Management of upper abdominal vascular trauma. Am J Surg. 1974;128(6):823-8.

12. Dukarm CP, Byrd RS, Auinger P, et al. Illicit substance use, gender, and the risk of violent behavior among adolescents. Arch Pediatr Adolesc Med. 1996;150(8):797-801.

13. Fagan J. Interactions among drugs, alcohol, and violence. Health Aff. 1993; 12(4):65-79.

14. Delmonte CAF. Vascular por balística terminal: lesão projétil de alta energia. [dissertação]. São Paulo (SP): Escola Paulista de Medicina; 1994.

15. Carvalho R, Severo G. A escalada da violência. A crítica. Manaus, 22 jan. 2000. p. C-1

16. Moore EE, Dunn EL, Moore JB, et al. Penetrating abdominal trauma index. J Trauma. 1981;21(6):439-45.

17. Costa OL, Faintuch J, Colombo CA, et al. Valor de um índice prognóstico anatômico na classificação de pacientes traumatizados com lesões abdominais e extra-abdominais. Rev Paul Med. 1988;106(3):131-5. 
18. Degiannis E, Velmahos GC, Levy RD, et al. Penetrating injuries of the abdominal inferior vena cava. Ann R Coll Surg Engl. 1996;78(6):485-9.

19. Ombrellaro MP, Freeman MB, Stevens SL, et al. Predictors of survival after inferior vena cava injuries. Am Surg. 1997;63(2):1478-83.

20. Klein SR, Baumgartner FJ, Bongard FS. Contemporary management strategy for major inferior caval injuries. J Trauma. 1994;37(1):35-42.

21. Coimbra R, Santos PE, Caffaro RA, et al. Traumatismo de veia cava inferior: análise de 36 casos. Rev Ass Med Bras. 1993;39(4):229-33.

22. Burihan E. Lesões vasculares iatrogênicas. In: Maffey FHA. Doenças vasculares periféricas. $2^{\mathrm{a}}$ ed. Rio de Janeiro: Medsi; 1995. p. 1127-42.

23. Leppaniemi AK, Savolainen HO, Salo JA. Traumatic inferior vena caval injuries. Scand J Thorac Cardiovasc Surg. 1994;28(34):103-8.

24. Rozycki GS. Ultra-sonografia para cirurgiões. In: Clínicas Cirúrgicas da América do Norte. Vol. 2. Rio de Janeiro: Interlivros; 1999.
25. Mullins RJ, Huckfeldt R, Trunkey DD. Abdominal vascular injuries. Surg Clin North Am. 1996;76(4):813-32.

26. Timberlake GA, Kerstein MD. Venous injury: to repair or ligate, the dilemma revisited. Am Surg. 1995;61(2):139-45.

27. Silva JCB, Dolnikoff MS, Moura LAR, et al. Ligation of the left renal vein in epm1-Wistar rats: functional and morphologic alterations in the kidneys, testes and suprarenal glands. São Paulo Med J. 1997;115(4):1475-84.

28. Wiencek RG, Wilson RF. Inferior vena cava injuries - the challenge continues. Am Surg. 1988;54(7):423-8.

29. Kudsk KA, Sheldon GF, Lim RC. Atrial-caval shunting (ACS) after trauma. J Trauma. 1982;22(2):81-85.

Endereço para correspondência:

Cleinaldo de Almeida Costa

Rua 24 de Maio, 220 / 719

Centro - Manaus - AM

CEP: 69010-080

E-mail: cleinaldocosta@uol.com.br 